\title{
Use of Rituximab in Refractory Dermatomyositis: A Case Report
}

\section{K. Grover, R. Peshin}

Department of Rheumatology, Nobles Hospital, Isle of Man, UK.

Email: rohit.peshin@gov.im, karangrover@outlook.com

Received December $4^{\text {th }}, 2012$; revised January $16^{\text {th }}$, 2013; accepted January $25^{\text {th }}, 2013$

\begin{abstract}
We report a case of a 56-year-old female with a confirmed diagnosis of Dermatomyositis on muscle biopsy, which was refractory to 5 different disease modifying drugs as well as intravenous immunoglobulins and symptoms improved dramatically with a single course of Rituximab. We hereby wish to highlight the importance of Rituximab in this highly resistant case and that anti CD 20 biologic drugs can be considered as standard treatment protocol in refractory Dermatomyositis.
\end{abstract}

Keywords: Dermatomyositis; Rituximab

\section{Case Report}

A 60 years old lady presented to the rheumatology service in November 2007, with one month history of generalised rash and increasing muscle fatigue. There was no significant past medical history and she was not taking any regular medications. She worked as an administrative clerk.

She initially noticed the itchy and scaly rash at back of scalp which soon spread over to face, chest and bilateral upper limbs. She noticed muscle weakness with difficulty to mobilise and get out of the bed and for activities of daily living.

Physical examination revealed maculopapular, erythematous, semi-calcified rash on her cheeks and her neck. She had a classical heliotrope rash as well as evidence of Gottron's papules. She had bilateral upper limb weakness in the proximal $(3 / 5)$ and distal $(4+/ 5)$ muscle groups as well as neck muscle weakness (3/5). She was afebrile and all vital signs were normal.

Routine blood tests showed creatine kinase (CK) level of 2799 (10 - 195 units/L). ANA was +ve at 1:320 but ENA, ANCA, Hepatitis screen were negative. CT chest, abdomen and pelvis did not show any evidence of malignancy. Biopsy of skin was consistence with Dermatomyositis.

She was initially treated by intravenous bolus of 1 gram methyprednisolone followed by tapering dose of prednisolone. As she was unable to wean off to less than $30 \mathrm{mg}$ of prednisolone after six months of treatment with frequent flare up symptoms, Azathioprine was introduced as steroid sparing drug.
She was again presented with severe flare up the rash and muscle weakness after 12 weeks of Azathioprine along with prednisolone $20 \mathrm{mg}$ OD. CK levels were with in the normal limits but hydroxybutarate dehydrogenase (HBDH) levels were elevated of 208 (72 - 182 units/L). Hydroxychloroquine was introduced for the cutaneous lesions, while continuing the Azathioprine and oral prednisolone. She unfortunately developed allergic reaction to Hydroxychloroquine requiring treatment with antihistaminic drugs.

Azathioprine was stopped due to inefficacy as skin rash was getting very active, burning and very itchy. She was started on Mycophenolate Mofetil (MMF) and dose was increased to 1 gram bd without much improvement. Decision was made for intravenous immunoglobulin infusions which she received over 5 days in May, 2009. Her symptoms did improve briefly and she was able to stop oral steroids completely for next 3 months.

She was readmitted in October, 2009 with dysphagia as well as proximal muscles and neck weakness. Muscle power was $3 / 5$ in bilateral upper limbs, $4+/ 5$ in lower limbs and 3/5 in neck muscles. CK, urine myoglobin, LFTs were normal but HBDH was elevated to 213. She was started on cyclosporine, the dose of which is built up to $200 \mathrm{mg}$ bd while continuing on $30 \mathrm{mg}$ of prednisolone (tapering dose).

Over the course of next year her symptoms did improve on cyclosporine and oral steroids though she was unable to wean off the dose of steroids to less than $10 \mathrm{mg}$ OD. Unfortunately her renal functions deteriorated which was thought to be secondary to cyclosporine and dose 
was reduced to $100 \mathrm{mg}$ bd. This resulted in another flare up of her condition. At this stage her activities of daily living were severely affected, and she had become wheel chair bound with proximal muscle weakness. She was very steroid sensitive and dependant on high dose steroids. The decision was taken to treat her with Rituximab.

She was subsequently treated with 2 doses of Rituximab $(2 \times 1$ gram $)$ infusions in starting February 2011 . Within 12 weeks post treatment, the rash and muscle weakness completely resolved. She was feeling better and was now able to safely reduce her steroid dosage.

At a recent clinical review 18 months post Rituximab therapy, her symptoms are well under control with no evidence of rash and muscle power of 5/5 in all muscle groups. Blood tests showed normal inflammatory markers, LFTs as well as CK. She had discontinued steroids now six months previously, with no evidence of recurrence of disease.

\section{Discussion}

Dermatomyositis is a subset of inflammatory idiopathic disorders [1] which are systemic connective tissue diseases defined by chronic muscle inflammation and weakness associated with autoimmunity [2]. It is a microangiopathy affecting skin and muscle; activation and deposition of complement causes lysis of endomysial capillaries and muscle ischaemia [3].

Standard treatment regimes include initial induction of remission with high dose intravenous steroids followed by tapering dose of prednisolone, and introduction of Disease Modifying Anti Rheumatic Drugs (DMARDS) e.g. Methotrexate and Azathioprine to maintain remission. Failure to achieving remission with glucocorticoids and two different DMARDS is generally considered to be refractory to treatment [4], although there are no set guidelines regarding this.

There are only few case reports of Rituximab treatment for recurrent and refractory Dermatomyositis [5-7]. Rituximab is fast emerging as primary treatment for refractory Dermatomyositis [8-10].

Our case report further establishes these credentials, as we had tried 5 different DMARDS along with high dose steroids, as well as intravenous immunoglobulins, but none seems to have brought the disease under control. Beside induction of remission, the beneficial effects and remission seems to be sustained as in our patient even after 18 months follow up. This is significant but further research needs to done with possible case control studies before Rituximab can be established as standard treat- ment for the refractory Dermatomyositis.

\section{REFERENCES}

[1] P. H. Plotz, M. Dalakas, R. L. Leff, L. A. Love, F. W. Miller and M. E. Cronin, "Current Concepts in the Idiopathic Inflammatory Myopathies: Polymyositis, Dermatomyositis, and Related Disorders," Annals of Internal Medicine, Vol. 111, No. 2, 1989, pp. 143-157.

[2] T. P. O’Hanlon, D. M. Carrick, I. N. Targoff, F. C. Arnett, J. D. Reveille, M. Carrington, X. Gao, C. V. Oddis, P. A. Morel, J. D. Malley, K. Malley, E. A. Shamim, L. G. Rider, S. J. Chanock, C. B. Foster, T. Bunch, P. J. Blackshear, P. H. Plotz, L. A. Love and F. W. Miller, "Immunogenetic Risk and Protective Factors for the Idiopathic Inflammatory Myopathies," Medicine (Baltimore), Vol. 85, No. 2, 2006, pp. 111-127. doi:10.1097/01.md.0000217525.82287.eb

[3] M. C. Dalakas and R. Hohlfeld, "Polymyositis and Dermatomyositis,” The Lancet, Vol. 362, No. 9388, 2003, pp. 971-982. doi:10.1016/S0140-6736(03)14368-1

[4] M. L. Miller and S. A. Rudnicki, "Treatment of Recurrent and Resistant Dermatomyositis and Polymyositis in Adults.”

http://www.uptodate.com/contents/treatment-of-recurrentand-resistant-dermatomyositis-and-polymyositis-in-adults ?source=search_result\&search=dermatomyositis\&selecte dTitle $=8 \% 7 \mathrm{E} 150$

[5] E. H. Noss, D. L. Hausner-Sypek and M. E. Weinblatt, "Rituximab as Therapy for Refractory Polymyositis and Dermatomyositis," The Journal of Rheumatology, Vol. 33, No. 5, 2006, pp. 1021-1026.

[6] E. A. Mahler, M. Blom, N. C. Voermans, B. G. van Engelen, P. L. van Riel and M. C. Vonk, "Rituximab Treatment in Patients with Refractory Inflammatory Myopathies,” Rheumatology (Oxford), Vol. 50, No. 12, 2011, pp. 2206-2213. doi:10.1093/rheumatology/ker088

[7] T. D. Levine, "Rituximab in the Treatment of Dermatomyositis: An Open-Label Pilot Study,” Arthritis \& Rheumatism, Vol. 52, No. 2, 2005, pp. 601-607. doi:10.1002/art.20849

[8] A. E. Hak, B. de Paepe, J. L. de Bleecker, P. P. Tak and M. de Visser, "Dermatomyositis and Polymyositis: New Treatment Targets on the Horizon,” Netherlands Journal of Medicine, Vol. 69, No. 10, 2011, pp. 410-421.

[9] V. Limaye, P. Hissaria, C. L. Liew and B. Koszyka, "Efficacy of Rituximab in Refractory Antisynthetase Syndrome,” Internal Medicine Journal, Vol. 42, No. 3, 2012, pp. e4-e7.

[10] I. Marie and L. Mouthon, “Therapy of Polymyositis and Dermatomyositis,” Autoimmunity Reviews, Vol. 11, No. 1, 2011, pp. 6-13. doi:10.1016/j.autrev.2011.06.007 